\title{
Téoros
}

Revue de recherche en tourisme

\section{Historique et évolution du tourisme en Estrie / Cantons-de-l'Est}

\section{Réjean Beaudoin}

Volume 16, numéro 1, printemps 1997

La région touristique de l'Estrie / Cantons-de-l'Est

URI : https://id.erudit.org/iderudit/1074919ar

DOI : https://doi.org/10.7202/1074919ar

Aller au sommaire du numéro

Éditeur(s)

Université du Québec à Montréal

ISSN

0712-8657 (imprimé)

1923-2705 (numérique)

Découvrir la revue

Citer cet article

Beaudoin, R. (1997). Historique et évolution du tourisme en Estrie /

Cantons-de-l’Est. Téoros, 16(1), 4-7. https://doi.org/10.7202/1074919ar d'utilisation que vous pouvez consulter en ligne.

https://apropos.erudit.org/fr/usagers/politique-dutilisation/ 


\section{than. \\ Historique et ÉVOLUTION DU TOURISME EN Estrie / Cantons-De-L'Est}

Réjean Beaudoin, conseiller expert en tourisme

Faire un historique et parler de l'évolution du tourisme en Estrie / Cantons de-l'Est $m$ 'est apparu une âche fort intéressante. Cette région, où je suis né et qui m' habite toujours, a suivi, elle aussi, les divers méandres de l'évolution touristique québécoise, mais avec des nuances qui la démarquent des autres régions du Québec.

Aussi, le long de cene randonnée, nous allons identifer la venue des premiers villegiateurs du XIXe siècle, jeter un regard sur la periode $1900-1965$, puis suivre celle de 1965 - 1980 pour terminer avec celle de 1980 à aujourd hui.

\section{LA VENUE DES LOYALISTES AMÉRICAINS}

Outre l'ère amérindienne, les premiers colons à s'établir dans les Cantons-deI'Est' furent des loyalistes américains qui y fondèrent de nombreuses agglomérations dont Sherbrooke (1791), Austin (1793), Stantead Plain (1796) ete. Leur venue a non seulement permis le développement économique de la région, mais aussi, la mise en place d'une vie culturelle, qui se perpetue encore de nos jours et qui distingue touristiquement la région d'autres milieux régionaux québécois.

\section{AU XIXE SIÈCLE}

Il semble admis que les débuts ${ }^{2}$ du tourisme dans les Cantons-de-l'Est remontent à 1850 , au moment de la fondation sur les bords du Lac Memphrémagog du *Hermitage Country Clubs par le juge Drummond de Montréal. En 1880, toute la rive s'échelonnant entre la ville de Magog et la localité de Georgeville était occupée par des villégiateurs provenant de Montréal, Sherbrooke, Boston et même New-York. Les activités sont souvent liées au monde aquatique (baignade, pêche, canotage) et la promenade dans la nature. Trois autres lacs de la région, le Magog, le Massawippi et le Brone ont vu émerger des hôtels et des villas le long de leurs rives. Des localités s'y développèrent comme Knowlton, North Hatley, Ayer's Cliff.

Puis, la venue du chemin de fer a rendu le tourisme un peu plus itinerant' :

*... the ligne reaches the prosperous and busy town of Sherbrooke, situated at the junction of the Magog and St-Francis Rivers, amidst a beautiful surrounding country. Near the village are the long Rapids of the Magog. The next point of interest on the route is Lennoxville ... .

\section{LA PÉRIODE DE 1900 À 1965}

Cette période est caractérisée par l'apparition de l'automobile et son côtoiement avec le chemin de fer, qu'elle finit par dominer nettement. L'intermodalité chemin de fer / cheval disparaît graduellement pour laisser la place à l'intermodalité chemin de fer / auto.

Les lieux d'activitês, d'hébergement, de restauration, etc., s'installent le long des routes à des points stratégiques ou gravitent autour des gares de chemin de fer. Selon la mode du temps, la promotion parle de lacs, de montagnes, des beautés du paysage, mais aussi de la force économique des Cantons-de-l'Est.

La région as son propre guide" de la route spécifiant les conditions routières, la population locale et leurs industries, des lieux d'hébergement, des stations-service, ete.

Au début des années 30, la promotion ${ }^{5}$ du gouvernement du Québec présente les Cantons-de-l'Est comme la wew England of Canadar. A la méme période, la *Cité de Sherbrooke* a un bureau de renseignements touristiques et compte 905 chambres d'hôtels', la Ville de Magog 66 et la ville de Granby 101.

Avec un meilleur système routier et l'automobile qui prend de plus en plus d'ampleur, les touristes doivent nécessairement passer dans les adowntown" pour se rendre al destination. Durant cette période, principalement après la deuxième guerre mondiale, de nombreux visiteurs, originant surtout de la Nouvelle-Angleterre, traversaient, pendant l'été et l'automne, plusieur's agglomérations des Cantons-de-l'Est pour se rendre à Québec, Montréal ou autres destinations estriennes ou québecoises. Les dollars américains circulaient. 


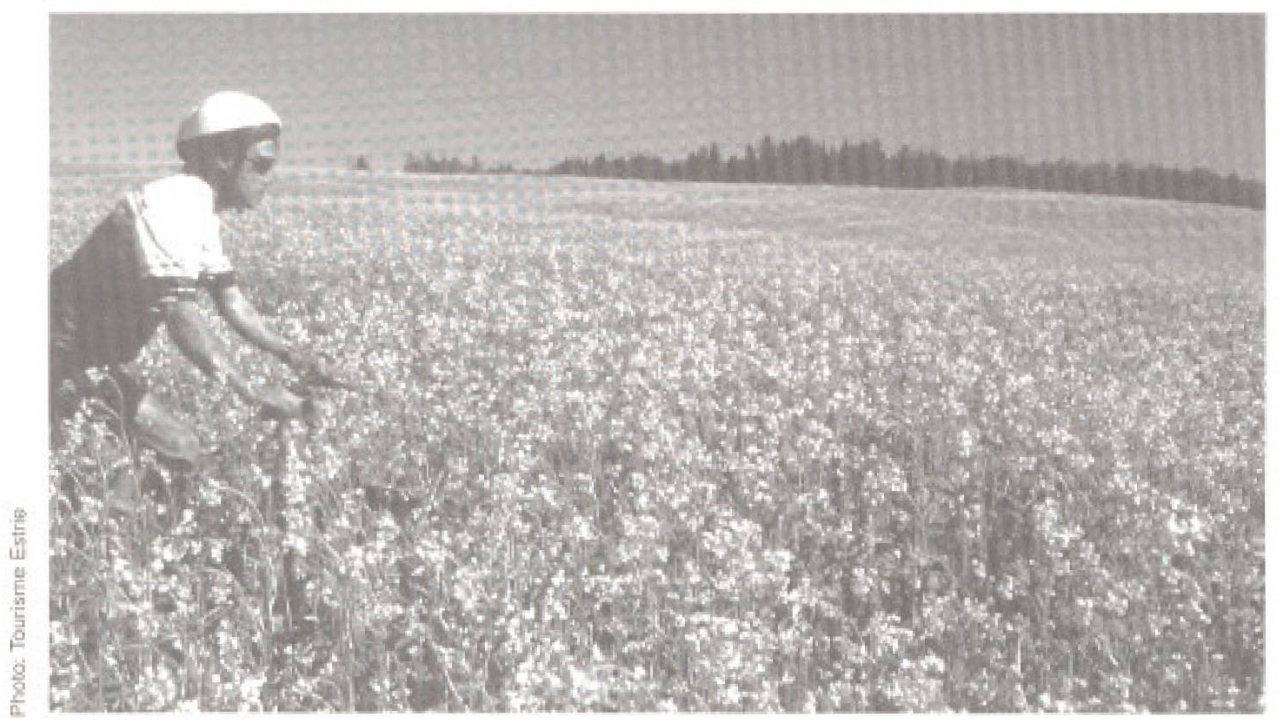

Les motels situés à l'entrée des villes ainsi que les hôtels, restaurants el commerces des centres-villes bénéliciaient grandement de ces touristes de passage. Les stations-service avaient le rôle principal de dispenser l'information et de distribuer les dépliants et les cartes routières.

Jusqu'à la fin des années 50 . les lieux de villégiature et les résidences secondaires sont fréquentés durant l'été et fermés durant les autres mois de l'année. Toutefois, à partir de la décennie 60 , avec le potentiel du skieur alpin/touriste, des centres de villégiature tel que Sutton, Magog Orford, North Hatley enclenchent la tradition d'une véritable saison touristique hivernale dans les Cantons-de-l'Est.

En termes de publicité, l'entreprise privilégie le dépliant et de petites annonces individuelles dans quelques journaux de Montréal et de la Nouvelle-Angleterre. Sauf dans le cas de $\alpha$ Ski dans l'Est $\%$, une entité regroupant des stations de ski alpin de la région, aucune concertation n'existe entre les intervenants. Quant à l'organisation du tourisme, quelques comités existent au sein de certaines chambres de commerce comme celle de Sherbrooke, mais curieusement composés de citoyens voués au développement de la collectivité, plutôt que d'intervenants touristiques dont la prospérité est perceptible.

L'inauguration de l'autoroute des Can-

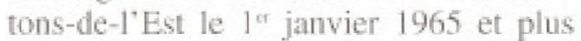
tard l'autoroute 55 sonnent la fin de cette période 1900 - 1965.

\section{LA PÉRIODE 1965 - 1980}

La mise en place d'autoroutes fut accueillie avec beaucoup de satisfaction. Mais une infrastructure de ce type change également les habitudes touristiques. Le facteur distance-temps rapprochait les Montréalais des Cantons-de-l'Est, alors que les Américains avaient maintenant un corridor leur permettant de traverser la région sans passer dans les villes pour se rendre à Montréal et plus tard à Québec.

De plus, comment une industrie touristique régionale, sans tradition de concertation, plus experte en tourisme de passage qu'en tourisme de destination, pouvait-elle relever le défi du changement?

Aussi, pendant longtemps, l'industrie touristique des Cantons-de-l'Est a peu réagi face à la nouvelle situation. Et ce manque de réaction, ce manque d'adaptation a produit les résultats suivants:

Relativement au marché de Montréal, les impacts sont ni plus ni moins automatiques ${ }^{\top}$ et se font sentir de cette façon:

plus de visiteurs d'une journée incluant 1 ou 2 visites touristiques et un repas dans un restaurant ou sous forme de pique-nique;

plus de villégiateurs ayant leur résidence secondaire:

une légère augmentation de lieux d'hébergement (Sutton, Magog Orford, Bromont);

le jardin zoologique de Granby est l'attrait le plus connu, le théatre d'êté
La Marjolaine et le Centre d'Arts d'Orford font aussi connaître le nilieu;

le ski alpin est l'activité hivernale la plus notoire et la plus articulée;

globalement, les Cantons-de-l'Est sont perçus comme une région de lacs et de montagnes habitée par des fran= cophones et des anglophones.

Moins de visiteurs utilisent graduellement la route 1 (aujourd'hui la route 112), ce qui a affecté de nombreux commerces d'hébergement, de restauration, etc. A partir de lá fin des années 50 et suivantes, aux Etats-Unis, lors de l'implantation d'autoroutes, les commerçants de routes principales, souvent parallèles aux autoroutes, mettaient sur pied une association de route afin de conserver le plus de clientèle possible ou favorisatent la création et la promotion de circuits touristiques visant le marché des automobilistes touristes. A titre d'exemples, on peut citer sl'Association de la route $30 \mathrm{l} w$ dans l'Est des Étants-Unis et la xincoln trailw dans le Midwest. Mais dans les Cantons-de-l'Est, sauf dans le cas du projet du circuit touristique Le triangle du Québecm à la fin des années 60, aucune réaction de ce genre n'a eu lieu et les touristes de passage québécois n'ont pas tous été remplacés par des touristes québécois de destination pendant cette période.

En ce qui concerne les touristes américains, le même changement s"est produit. Au lieu de continuer à voyager sur la route 5 (aujourd'hui 143), progressivement, les Américains se rendaient à Montréal et à Québec via l'autoroute 55. Evitant les centres-villes parce que ne sachant pas suffisamment ce qui s'y trouvait, de nombreux commerçants établis le long de l'ancienne route, dans le centreville de Sherbrooke et autres endroits ont été touchés. Restaurants, hôtels, stations= service, etc. ont subi le rude contre-coup de ce changement.

Un comité touristique existe à Sherbrooke, la région de Magog a une association, Granby a un office de tourisme. alors que certaines chambres de com= merce ont un comité voué au secteur tourisme. Malgré leurs efforts, les difficultés persistent. 
Vers 1975 - 1976, de la prosperité, beaucoup sont passés à la stagnation et à la fermeture. Toutefois, si une meilleure expertise touristique - ils avaient l'expertise hoteliere, en restauration, etc. mais pas en tourisme _ avait caractêrisée le milieu, l'industrie touristique du temps aurait ếté en mesure de rencontrer avec plus d'adéquation ce grand defi en se concertant mieux et plus, en faisant de la publicité collective et en croyant à la formation continue.

Aussi, à la fin des années 70, les Cantons= de-l'Est étaient une région touristique déclinante, non organisée, nantie d'un faible nombre d'intervenants qui se concertaient peu, sauf dans le domaine du ski alpin, sans stratégie de marketing, préoccupée par la mise en place de bureaux d'information touristique.

Face à une telle situation, des éléments dynamiques de la région, sensibilisés sur limportance et l'avenir du tourisme, songent à la régionalisation touristique. Ils croient au potentiel, mais pour en bénéficier, les Cantons-de-l'Est doivent se promouvoir et se développer régionalement parce que les touristes voyagent régionalement. Grâce au support du Conseil régional de développement de l'Estrie et du ministère du tourisme du Québec, la régionalisation touristique devient un sujet de plus en plus d'actualité.

\section{LA PÉRIODE 1980 À AUJOURD'HUI}

Après une année de démarches et de sensibilisation, l'Association touristique de l'Estric (ATE) est ofliciellement fondéc à Richmond en 1979. Le premier président a été le regretté Monsieur Jean-Louis Dupont, alors maire de la ville de Rock Island.

A cette période, les intervenants en tourisme des Cantons-de-l'Est, n'étaient pas en nombre suffisants pour former un organisme touristique régional. Aussi, à ses débuts, le monde municipal et scolaire étaient plus présents que les intervenants en tourisme.

Pour bien grandir et combler un retard. les Cantons-de-1'Est touristiques devaient immédiatement s'occuper du développement du produit et faire en même temps de la promotion. Avec une force de plus de 52 municipalités membres et des intervenants en tourisme, de toutes les régions touristiques du Québec, les Cantons-de-l'Est étaient probablement les seuls à penser développement et promotion, tandis que les autres régions pensaient plus promotion.

Le grand départ du développement a été le projet de la Gorge de Coaticook. C'est d'ailleurs avec ce dossier que l'ere du tourisme moderne a pris son envol dans les Cantons-de-l'Est. Par la suite, bien d'autres dossiers ont été entrepris et réalisés: l'agrandissement du Pare du Mont Orford, l'expansion de plusieurs stations de ski alpin, la côte magnétique de Chartierville, l'aménagement de la Baie des sables de Lac Mégantic, le Parc de récréation de Frontenac, le projet d'implantation du premier casino québecois dans la région Magog-Orford (1983), la confection et la promotion d'une fine cuisine estrienne, etc. La région avait une effervescence telle en termes de développement touristique que, lors du Sommet socio-économique de l'Estrie qui a eu lieu en janvier 1985 , le secteur du tourisme a ravi près de 35 millions de dollars en engagements gouvernementaux, soit la plus grande part sectorielle de 1'evenement. Ainsi, les Cantons-del'Est se garnissaient d'attraits et d'activités pour renforcer sa puissance attractive.

Sur le plan de la promotion, ce fut aussi un grand départ. II aura fallu deux mois pour se concerter et ćlaborer la première demi page de publicité coopérative dans le quotidien La Presse de Montréal. Les intervenants ayant eu de bons résultats, il a été par la suite assez aisé de consacrer cette tradition indispensable qui se poursuit. Afin de se distinguet des autres régions et pénétrer mieux les marchés cibles, les Cantons-de-l'Est s'étaient dotés d'une image de marque mettant en relief la culture et la nature en Estrie. Cette image était reproduite en quatre couleurs sur la carte routière régionale, l'affiche, le guide touristique d'alors ainsi que dans les pages publicitaires collectives. Cette approche a eu un remarquable impact, puisque la dite image de marque était reproduite des milliers et des milliers de fois.

Ainsi, avec une activité fébrile sur les plans du développement et de la promo- tion, vers 1985 et durant les suivantes, les Cantons-de-l'Est s'étaient sculptés une place de choix au sein de l'échiquier touristique québecois.

En 1987 - 1988, la région s'est dotée d'un plan de développement touristique qui est révisé périodiquement, alors que le marketing est dynamise par la woie d'un plan stratégique triennal. A divers degrés. Tourisme Estrie fait maintenant de la promotion au Québec, aux États-Unis et en France. Depliants, brochure de forlaits, affiches, présence à des salons, publicités coopératives, etc., font partie des moyens destines à faire connaître l'Estrie / Cantons-de-l'Est à ces marchés. Sur le plan du développenent, l'organisme s'est hautement impliqué dans la mise en valeur d'une piste cyclable panrégionale agencé à celle des régions limitrophes.

Des 20 millions de dollars de recettes touristiques en 1979, les Cantons-de1'Est retirent en 1994, près de 193.8 millions de revenus touristiques ou 9,7 fois plus:. Une récente extrapolation prévoit des recettes de l'ordre de 214,2 millions de dollars en 1998.

De ses quelques villegiateurs du XIXe siècle, en passant par une prospérité découlant du tourisme de passage américain dans les villes et villages, en passant par un virage dramatique issu de la mise en place d'autoroutes qui ont change les habitudes touristiques, en passant du tourisme estival à un tourisme quasi quatre saisons, en adoptant graduellement la concertation et la régionalisation touristiques, en se préoccupant de développement, en se dotant d'une stratégie permanente de mise en marché, les Cantons-del'Est touristiques ont contourné bien des méandres pour permettre aujourd'hui à plus de 11000 Estriens et Estriennes de bénéficier directement ou indirectement de l'industrie du tourisme.

Nonobstant cette ascendance, les Cantons-de-l'Est doivent toujours avoir à l'esprit que la compétition est plus que presente, que la formation continue est urgente, qu'il ne faut pas dire que wça va bien mais plutôt dire sça doit aller mieuxw, qu'il faut penser région, mais aussi interrégion, qu'il faut maintenir la qualité de vie et demeurer la vraie région des Cantons-de-l'Est avec sa culture et sa nature d'avenir. 


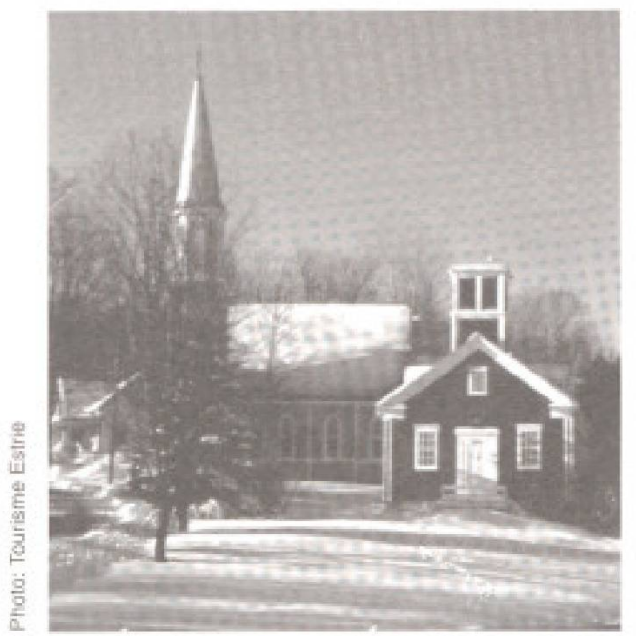

\section{ET LE XXIE SIĖCLE}

Que seront les Cantons-de-l'Est touristiques au XXIe siècle? Même si la réponse à une telle question n"est pas évidente, je me permets toutefois un peu de prospective.

Pour mol, ce sera l'ère "Ecosium " à-dire une sorte de dialogue, une sorte d'échange profond entre les touristes et l'environnement qu'ils visitent. Ce seral le siècle de la restauration de la planète. Beaucoup de programmes de vacances spécifiques seront élaborés à cet effet.

Aussi, les Cantons-de-l'Est seront plus verts qu'aujourd'hui, le milieu rural sera populaire et reprendra une bonne partic de son authenticité. Le transport en commun revivia au détriment de l'satomobile. La grande mode sera l'etude de la nature et les différentes interrelations de ses composantes. Pour la chasse par exemple, les armes seront simples afin que l'être humain redécouvre ses talents naturels en cette matière. L'intérêt pour l'histoire sera importante dont celle des Amérindiens.

Ce scénario se réalisera-t-il? La parole appartient maintenant à la personne qui rédigera l'article sur l'historique et l'évolution du tourisme dans les Cantons-del'Est dans l'édition spéciale de la revue Téoros de l'an 2097.

\section{NOTES}

1 A l'époque, la région était désignée sous le vocable de The Eastern Townships.

2 Tiré du texte de BRIÊRE. Rogger (1967). *Tourisme", Bulletin de I"Assaciation des geographes de I'Amerique frangarise, no 11 , septembre.

3 GRAND TRUNK RAILWAY (1892), The Tourist Route of America.

4 PLANCHE, C.C. (1920), Planche"s Com. mercial Handbook and Road Guide of the Eastern Tounships, The Chronicle Printing Co, Cookshire.

5 MINISTĖRE DE LA VOIRIE (1930). The Eastern Townships of Quebec.

6 SERVICE HOTELILRIES (1930), Guide officiel - prix des chambres dans les hotel. leries, Province de Québec, première édition.

7 Malgré la venue des autoroutes, la région n'avait pass clatwore de stratégic de promotion.

8. Source : TOURISME QUÉBEC

9 ZINS BEAUCHESNE ET ASSOCIÉS (1995). Plan strarégigae de markeling 1995 1998. Association touristique de l'Estrie. octobre.

10 Sigle originant des mots beologie er symposium.

\section{TÉOROS VOUS PRÉSENTE SA COLLECTION COLLOQUES ET CONGRĖS:}

\section{UN AUTRE TRAIT D'UNION ENTRE LE MONDE DE LA RECHERCHE ET CELUI DES PROFESSIONNELS}

Cette nouvelle collection réunit les textes des conférences et des communications prononcées lors de différents colloques nationaux et internationaux qui ont eu lieu au Québec depuis 1991.

Ainsi, chaque numéro vous permet de connaitre, sur un sujet donnè, ce qui se dit et ce qui se fait ici et ailleurs... et ce que des spécialistes et des intervenants de divers horizons et de diverses régions en pensent....

\section{Deja parus}

No 1: Les politiques touristiques $\left(34 \mathrm{p}_{\mathrm{u}}+\right.$ cartes).............................. 95

No 2: Premier colloque national sur le tourisme de eireuit $(44$ p. $\ldots \ldots \ldots \ldots \ldots$. s $\mathrm{s}$

No 3: Colloque international uFleuves et planètes $\{52 \mathrm{p} . \ldots \ldots \ldots \ldots \ldots \ldots \ldots \ldots$ g $\$$

No 4: Colloque national sur l'écotourisme $\mid 58 \mathrm{p}.\} \ldots \ldots \ldots \ldots \ldots \ldots \ldots \ldots \ldots \ldots$. 95

No 5: Colloque wTourisme et eulturew, une allianee d'avenir? $(48 \mathrm{p}.) \ldots \ldots \ldots \ldots$ is 5

No 6: Premier Colloque national sur le golf $(46$ p. $) \ldots \ldots \ldots \ldots \ldots \ldots \ldots \ldots$ s $\$$

No 7: Troisième colloque uÉcole sans frontieress

Le tourisme educatif et les besoins des aines $(200 \mathrm{p}.) \ldots \ldots \ldots \ldots \ldots \ldots$

No 8: Colloque sur Irecoutourisme fOuest du Québeci $(68 p.) \ldots \ldots \ldots \ldots \ldots \ldots$ is

No 9 Colloque québécois sur le tourisme d'aventure:

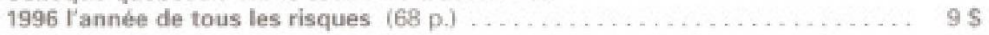

No 10: Premier congrès de la Fèdération québécoise

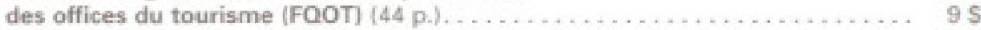

No 11: Colloque sur l'agrotourisme (a venir)................................. 9 \$ Les prix incluent les taxes

\section{Appel d'inscription dans le BOTTIN TOURISTIQUE 1997}

La $3^{2}$ édition de ce bottin sera publiée en avril-mai prochain, Cet annuaire, qui est diffusé a plus de 2500 exemplaites au Québec. au Canada et at l'étranger, fournit les coordonnées postales et téléphoniques, secteur d'activité et divers renseignements sur les intervenants du tourisme culturel, récréatif et d'affaires au Québec. Les frails d' inscription sont de $55 \$$, taxes comprises. Votre inscription doit toutefois nous parvenir dans les meilleurs délais, Pour \& inscrire ou pour obtenir de plus amples renseignements. s'adresser à:

Québec dans le monde

Teléphone: (418) 659-5540

Telécopieur: (418) 659-4143.

Email: quebecmonde@ total.net 